See discussions, stats, and author profiles for this publication at: https://www.researchgate.net/publication/274510153

\title{
Wavelet based flickering flame detector using differential PIR sensors
}

Article in Fire Safety Journal · December 2012

DOI: 10.1016/j.firesaf.2012.06.006

\section{CITATIONS}

25

7 authors, including:

Fatih Erden

North Carolina State University

50 PUBLICATIONS 307 CITATIONS

SEE PROFILE

Kivanc Kose

Memorial Sloan Kettering Cancer Center

80 PUBLICATIONS 543 CITATIONS

SEE PROFILE
READS

79

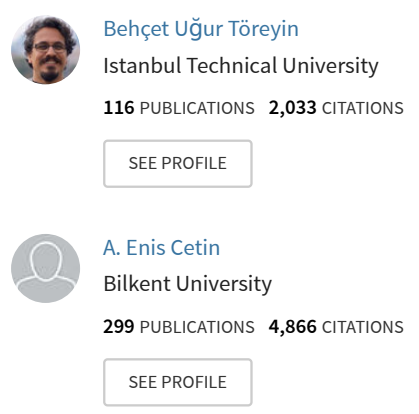

Some of the authors of this publication are also working on these related projects:

Grading of Cervical Cancer Histopathological Images View project

Automated Image Guidance for Diagnosing Skin Cancer with Confocal Microscopy View project 


\title{
Wavelet based flickering flame detector using differential PIR sensors is
}

\author{
Fatih Erden $^{\mathrm{a}, *}$, B. Ugur Toreyin ${ }^{\mathrm{b}}$, E. Birey Soyer ${ }^{\mathrm{c}}$, Ihsan Inac ${ }^{\mathrm{d}}$, Osman Gunay ${ }^{\mathrm{c}}$, \\ Kivanc Kose ${ }^{\mathrm{d}}$, A. Enis Cetin ${ }^{\mathrm{d}}$ \\ ${ }^{a}$ Department of Electrical and Electronics Engineering, Hacettepe University, Ankara, Turkey \\ ${ }^{\mathrm{b}}$ Department of Electronic and Communication Engineering, Cankaya University, Ankara, Turkey \\ ' ASELSAN Inc., Ankara, Turkey \\ ${ }^{\mathrm{d}}$ Department of Electrical and Electronics Engineering, Bilkent University, Ankara, Turkey
}

\section{A R T I C L E I N F O}

\section{Article history:}

Received 7 July 2011

Received in revised form

9 April 2012

Accepted 5 June 2012

Keywords:

Flame detection

Pyro-electric Infrared (PIR) sensor

Wavelet transform

Markov models

\begin{abstract}
A B S T R A C T
A Pyro-electric Infrared (PIR) sensor based flame detection system is proposed using a Markovian decision algorithm. A differential PIR sensor is only sensitive to sudden temperature variations within its viewing range and it produces a time-varying signal. The wavelet transform of the PIR sensor signal is used for feature extraction from sensor signal and wavelet parameters are fed to a set of Markov models corresponding to the flame flicker process of an uncontrolled fire, ordinary activity of human beings and other objects. The final decision is reached based on the model yielding the highest probability among others. Comparative results show that the system can be used for fire detection in large rooms.
\end{abstract}

(c) 2012 Elsevier Ltd. All rights reserved.

\section{Introduction}

Conventional point smoke and fire detectors typically detect the presence of certain particles generated by smoke and fire by ionization or photometry. An important weakness of point detectors is that the smoke has to reach the sensor. This may take significant amount of time to issue an alarm and therefore it is not possible to use them in open spaces or large rooms. The main advantage of differential Pyro-electric Infrared (PIR) based sensor system for fire detection over the conventional smoke detectors is the ability to monitor large rooms and spaces because they analyze the infrared light reflected from hot objects or fire flames to reach a decision.

An uncontrolled fire in its early stage exhibits a transition to chaos due to the fact that combustion process consists of nonlinear instabilities which result in transition to chaotic behavior via intermittency [2-5]. Consequently, turbulent flames can be characterized as a chaotic wide band frequency activity. Therefore, it is not possible to observe a single flickering frequency in the light spectrum due to an uncontrolled fire. In fact, we obtained a time

\footnotetext{
An earlier version of this study was presented in Turkish in part at the IEEE 16th Signal Processing, Communication and Applications Conference, SIU-2008 [1].

Corresponding author. Tel.: +90 312290 1525; fax: +90 3122664126 . E-mail addresses: erdenfatih@gmail.com (F. Erden),

toreyin@cankaya.edu.tr (B.U. Toreyin), bireysoyer@gmail.com (E.B. Soyer),

ihsaninac@gmail.com (I. Inac),gunayosman@gmail.com (O. Gunay)

kkoseug@gmail.com (K. Kose), enis4cetin@gmail.com (A.E. Cetin).
}

series from the sampled read-out signal strength values of a PIR sensor with flickering flames in its viewing range (cf. Fig. 3). It is clear from Fig. 3 that there is no single flickering frequency and that flame flicker behavior is a wide-band activity covering $1-13 \mathrm{~Hz}$. It is also reported in the literature that turbulent flames of an uncontrolled fire flicker with a frequency of around $10 \mathrm{~Hz}$ [6,7]. Actually, instantaneous flame flicker frequency is not constant, rather it varies in time. Recently developed video based fire detection schemes also take advantage of this fact by detecting random high-frequency behavior in flame colored moving pixels [8-10]. Therefore, a Markov model based modeling of flame flicker process produces more robust performance compared to frequency domain based methods. Markov models are extensively used in speech recognition systems and in computer vision applications [11-14].

In $[15,16]$, several experiments on the relationship between burner size and flame flicker frequency are presented. Recent research on pyro-IR based combustion monitoring includes [17] in which monitoring system using an array of PIR detectors is realized.

A regular camera or typical IR flame sensors have a fire detection range of $30 \mathrm{~m}$. The detection range of an ordinary low-cost PIR sensor based system is $10 \mathrm{~m}$ but this is enough to cover most rooms with high ceilings. Therefore, PIR based systems provide a cost-effective solution to the fire detection problem in relatively large rooms as the unit cost of a camera based system or a regular IR sensor based system is in the order of one thousand dollars.

In the proposed approach, wavelet domain signal processing methods are used for feature extraction from sensor signals. 
This provides robustness against sensor signal drift due to temperature variations in the observed area. Notice that, differential PIR sensors are sensitive only to the changes in the intensity of the IR radiation within the viewing range rather than the absolute infrared radiation. In a very hot room the differential PIR sensor does not measure the temperature of the room, it only produces a constant output value which is not related with the temperature value. Regular temperature changes in a room are slow variations compared to the moving objects and flames. Since wavelet signals are high-pass and band-pass they do not get affected by slow variations in sensor signal.

There are two different classes of events defined in this approach. The first class represents fire events whereas the second class represents non-fire events. Each class of events is modeled by a different Markov model. The main application of PIR sensors is hot body motion detection. Therefore, we include regular human motion events like walking or running in the non-fire event class.

In Section 2, we present the operating principles of PIR sensors and how we modified the PIR circuit for flame detection. In Section 3, the wavelet domain signal processing and the Markov based modeling of the flames and human motion are described. In Section 4, comparative experimental results with other sensing modalities are presented.

\section{Operating principles of a PIR sensor system and data acquisition}

The main motivation of using a PIR sensor is that it can reliably detect the presence of moving bodies from other objects. Basically, it detects the difference in infrared radiation between the two 'segments' in its viewing range. Sensing normal variations in temperature and also disturbances in airflow are avoided by the elements connected in pairs. When these elements are subject to the same infrared radiation level, they generate a zero-output signal by canceling each other out [18]. Therefore, a PIR sensor can reject false detections accurately. The block diagram of a typical differential PIR sensor is shown in Fig. 1. A single sensor system requires additional expensive IR filters to distinguish ordinary hot bodies from $\mathrm{CO}$ and $\mathrm{CO}_{2}$ emissions. In this article, we show that it is possible to distinguish the flames from other hot bodies by analyzing the motion information captured by the differential system.

Commercially available PIR motion-detector read-out circuits produce binary outputs. However, it is possible to capture a continuous time analog signal indicating the strength of the

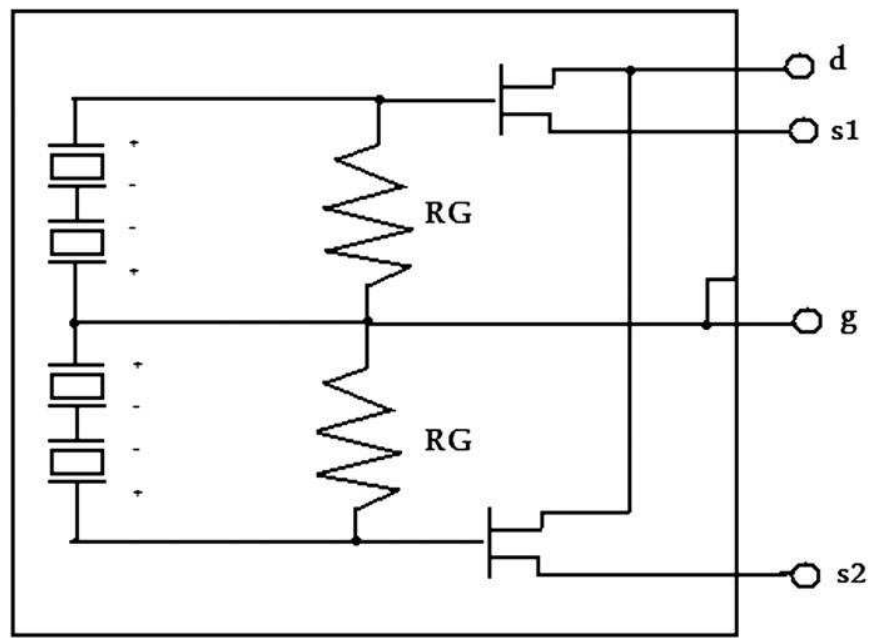

Fig. 1. The model of the internal structure of a PIR sensor received signal in time. The circuit diagram of a typical PIR motion-detector is shown in Fig. 2. It is possible to capture an analog signal from this circuit.

The circuit consists of four operational amplifiers (op. amps.), IC1A, IC1B, IC1C and IC1D. IC1A and B constitutes a two stage amplifier circuit whereas IC1C and D couple behaves as a comparator. The very-low amplitude raw output at the 2nd pin of the PIR sensor is amplified through the two stage amplifier circuit. The amplified signal at the output of IC1B is fed into the comparator structure which outputs a binary signal, either $0 \mathrm{~V}$ or $5 \mathrm{~V}$. Instead of using binary output in the original version of the PIR sensor read-out circuit, we directly capture the analog output signal at the output of the $2 \mathrm{nd} \mathrm{op}$. amp. IC1B and transfer it to a computer or a digital signal processor for further processing. The goal is to distinguish the flame signal from other signals due to ordinary moving bodies.

In uncontrolled fires, flames flicker. Following the discussion in Section 1 regarding the turbulent wide band activity of the flame flicker process, the analog signal is sampled with a sampling frequency of $f_{s}=50 \mathrm{~Hz}$ because the highest flame flicker frequency is $13 \mathrm{~Hz}$ and $f_{s}=50 \mathrm{~Hz}$ is well above the Nyquist rate, $2 \times 13 \mathrm{~Hz}$ [7]. In Fig. 3 , a frequency distribution plot corresponding to a flickering flame of an uncontrolled fire is shown. It is clear that the sampling frequency of $50 \mathrm{~Hz}$ is sufficient. Typical sampled signal for no activity case using 8 bit quantization is shown in Fig. 4. Other typical received signals from a moving person and flickering fire are presented in Figs. 5 and 6, respectively.

The strength of the received signal from a differential PIR sensor increases when there is motion due to a hot body within its viewing range. In fact, this is due to the fact that pyro-electric sensors give an electric response to a rate of change of temperature rather than temperature itself. On the other hand, the motion may be due to human motion taking place in front of the sensors or flickering flame. In this paper the differential PIR sensor data is used to distinguish the flame flicker from the motion of a human being like running or walking. Typically the PIR signal frequency of oscillation for a flickering flame is higher than that of PIR signals caused by a moving hot body. In order to keep the computational cost of the detection mechanism low, we decided to use Lagrange filters for obtaining the wavelet transform coefficients as features instead of using a direct frequency approach, such as the FFT based methods.

\section{Sensor data processing and Markov models}

Two different Markov models corresponding to flames and other motion are trained using the wavelet transforms of PIR recordings. Training of Markov models are carried out using various fire and motion recordings. During testing, the sensor signal is fed to both Markov models and the model producing the highest probability determines the class of the signal.

There is a bias in the PIR sensor output signal which changes according to the room temperature. Wavelet transform of the PIR signal removes this bias. Let $x[n]$ be a sampled version of the signal coming out of a PIR sensor. Wavelet coefficients obtained after a single stage subband decomposition, $w[k]$, corresponding to [ $12.5 \mathrm{~Hz}$ and $25 \mathrm{~Hz}$ ] frequency band information of the original sensor output signal $x[n]$ are evaluated with an integer arithmetic high-pass filter corresponding to Lagrange wavelets [19] followed by decimation. The filter bank of a biorthogonal wavelet transform is used in the analysis. The low-pass filter has the transfer function:

$H_{l}(z)=\frac{1}{2}+\frac{1}{4}\left(z^{-1}+z^{1}\right)$ 


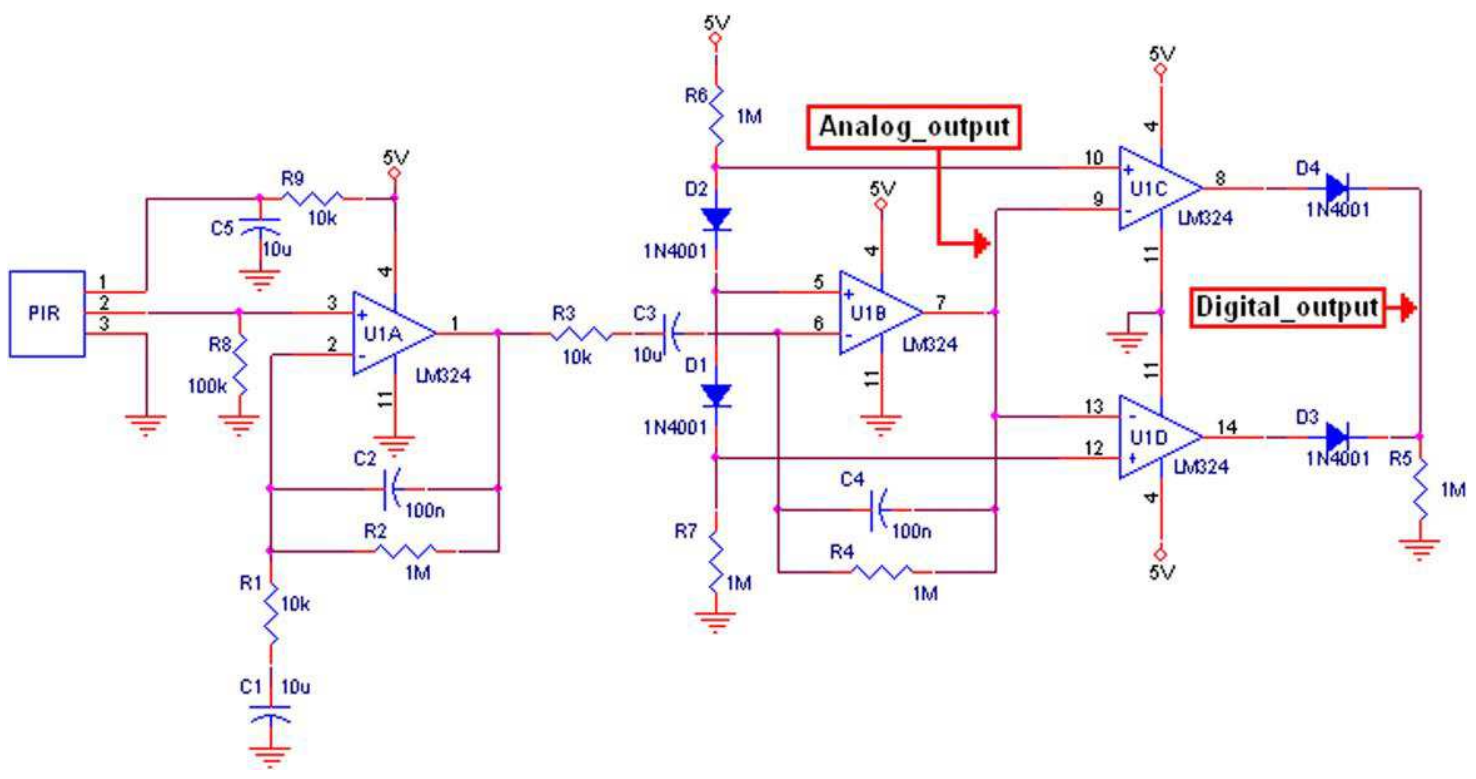

Fig. 2. The circuit diagram for capturing an analog signal output from a PIR sensor.

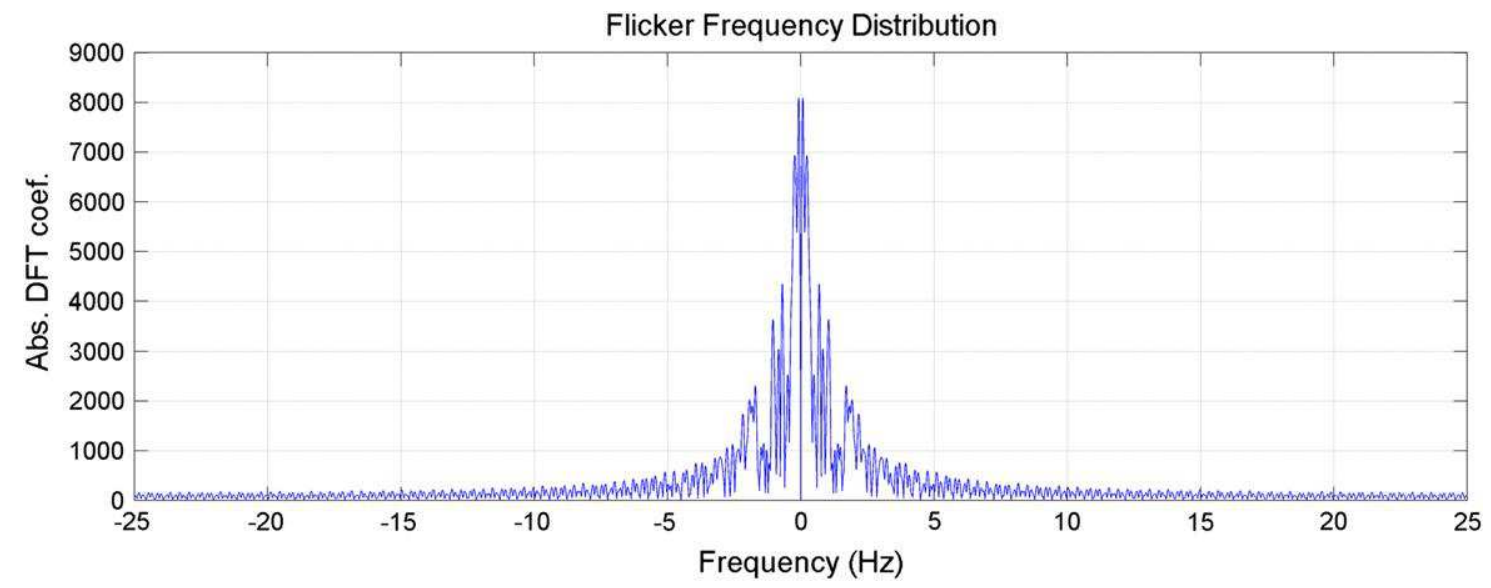

Fig. 3. Flame flicker spectrum distribution. PIR signal is sampled with $50 \mathrm{~Hz}$.

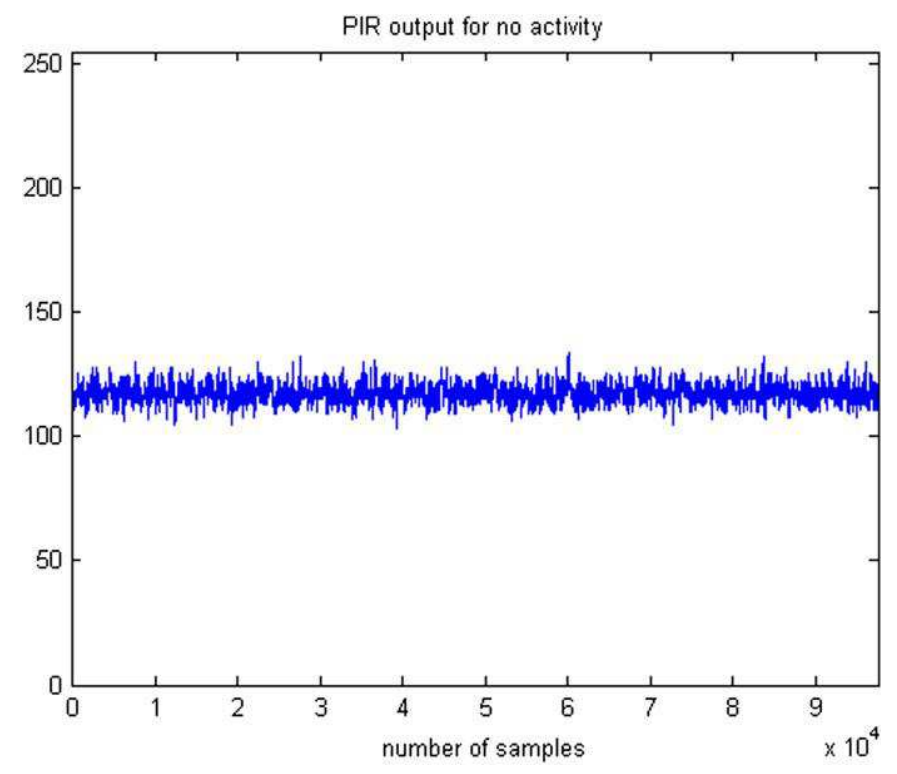

Fig. 4. A typical PIR sensor output sampled at $50 \mathrm{~Hz}$ with 8 bit quantization when there is no activity within its viewing range. and the corresponding high-pass filter has the transfer function

$H_{h}(z)=\frac{1}{2}-\frac{1}{4}\left(z^{-1}+z^{1}\right)$

A Markov model based classification is carried out for fire detection. Two three-state Markov models are used to represent fire and non-fire events (cf. Fig. 7). In these Markov models, state $S 1$ corresponds to no activity within the viewing range of the PIR sensor. The system remains in state $S 1$ as long as there is not any significant activity, which means that the absolute value of the current wavelet coefficient at index number $k,|w[k]|$, is below a non-negative threshold $T$, where $T$ is initialized based on the background noise level. This value can be estimated by a geneticalgorithm based approach, as in [20]. The second state, S2, which corresponds to an increase, or "rise" in consecutive wavelet coefficient values, is attained when

$w[k]-w[k-1]>T$

is satisfied. Similarly, the third state, $S 3$, which corresponds to a decrease, or "fall" in consecutive wavelet coefficient values, is attained when

$w[k]-w[k-1]<T$

is satisfied. 


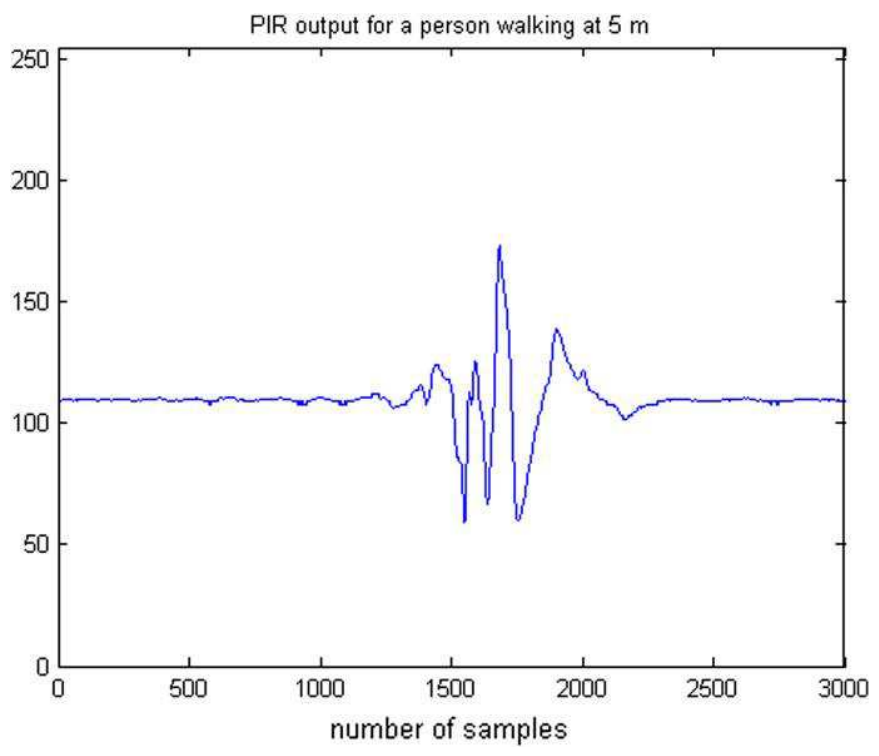

Fig. 5. PIR sensor output signal recorded at a distance of $5 \mathrm{~m}$ for a walking person.

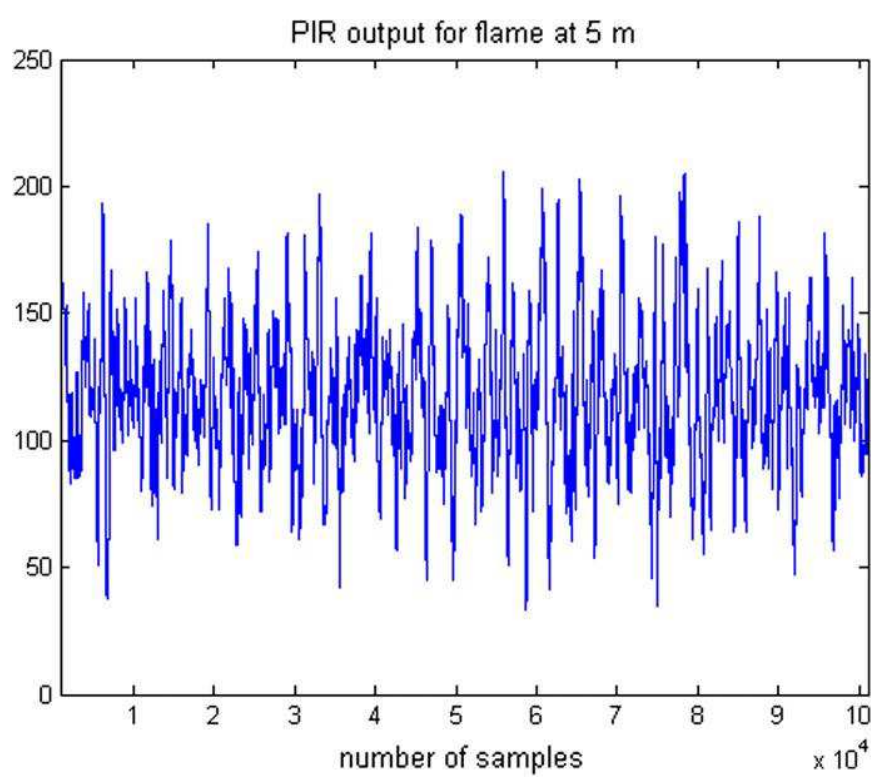

Fig. 6. PIR sensor output signal recorded at a distance of $5 \mathrm{~m}$ for a flame of an uncontrolled fire.

The first step of the Markov based analysis consists of dividing the wavelet coefficient sequences in windows of 25 samples. For each window, a corresponding state transition sequence is determined. An example state transition sequence of size 5 may look like

$C=\{S 2, S 1, S 3, S 2, S 1\}$

Since the wavelet signal captures the high frequency information in the signal, we expect that there will be more transitions occurring between states when monitoring fire compared to human motion.

The threshold $T$ in the wavelet domain determines the state transition probabilities, given a signal. In the training step, given $T$, ground-truth fire and non-fire wavelet training sequences, the task is to estimate the transition probabilities for each class. Let $a_{i j}$ denote the transition probabilities for the 'fire' class and $b_{i j}$ denote the transition probabilities for the 'non-fire' class. a

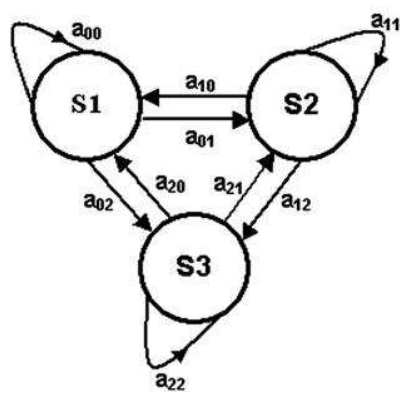

b

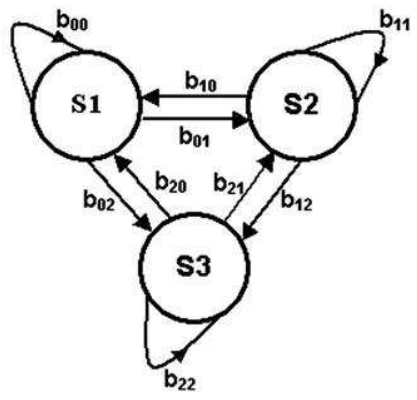

Fig. 7. Two three-state Markov models are used to represent (a) 'fire' and (b) 'nonfire' classes.

The decision about the class affiliation of a state transition sequence $C$ of size $L$ is made by calculating the two joint probabilities $P_{a}(C)$ and $P_{b}(C)$ corresponding to fire and non-fire classes, respectively

$P_{a}(C)=\prod_{i} p_{a}\left(C_{i+1} \mid C_{i}\right)=\prod_{i} a_{C_{i}, C_{i+1}}$,

and

$P_{b}(C)=\prod_{i} p_{b}\left(C_{i+1} \mid C_{i}\right)=\prod_{i} b_{C_{i}, C_{i+1}}$

where $p_{a}\left(C_{i+1} \mid C_{i}\right)=a_{C_{i}, C_{i+1}}, p_{b}\left(C_{i+1} \mid C_{i}\right)=b_{C_{i}, C_{i+1}}$, and $i=1, \ldots, L$.

In case of $P_{a}(C)>P_{b}(C)$ the class affiliation of state transition sequence $C$ will be declared as 'fire', otherwise it is declared as 'non-fire'.

For the training of the Markov models, the state transition probabilities for human motion and flame are estimated from 250 consecutive wavelet coefficients covering a time frame of $10 \mathrm{~s}$.

During the classification phase a state history signal consisting of 50 consecutive wavelet coefficients are computed from the received sensor signal. This state sequence is fed to fire and nonfire Markov models for each window. The model yielding the highest probability is determined as the result of the analysis of PIR sensor data.

For flame sequences, the transition probabilities $a$ 's should be high and close to each other due to random nature of uncontrolled fire. On the other hand, transition probabilities should be small in constant temperature moving bodies like a walking person, because there is no change or little change in sensor signal values. Hence, we expect a higher probability for $b_{00}$ than any other $b$ value in the non-fire model which corresponds to higher probability of being in $S 1$. The states $S 2$ and $S 3$ aims at tracking the rising and falling trends of the sensor signal in wavelet domain, respectively. Therefore, we expect frequent transitions between these states for uncontrolled fire.

\section{Experimental results}

The analog output signal is sampled with a sampling frequency of $50 \mathrm{~Hz}$ and quantized at 8bits. Real-time analysis and classification methods are implemented with $\mathrm{C}++$ running on a PC. Digitized output signal is fed to the PC via RS-232 serial port. It is also possible to process this signal using an FPGA or a digital signal processor.

In our experiments, we recorded fire and non-fire sequences at a distance of $5 \mathrm{~m}$ to the sensor. For fire sequences, we burned paper and alcohol, and recorded the output signals. For the nonfire sequences, we recorded walking and running person sequences. The person within the viewing range of the PIR sensor 
walked or ran on a straight line which was tangent to the circle with a radius of $5 \mathrm{~m}$ and the sensor being at the center.

The training set consists of 90 fire and 90 non-fire recordings with durations varying between 3 and $4 \mathrm{~s}$. The test set for fire class is 220 and that of non-fire set is 593. Our method successfully detected fire for 217 of the sequences in the fire test set. It did not trigger fire alarm for any of the sequences in the non-fire test set. This is presented in Table 1 .

The false negative alarms, 3 out of 220 fire test sequences, were issued for the recordings where a man was also within the viewing range of the sensor along with a fire inside a waste-bin. The test setting for which false alarms are issued is shown in Fig. 8. The system was tested live in a trade show for 3 days in Valladolid, Spain in November 2010. This was a FIRESENSE project activity [21]. The flame detector did not produce any false alarms and it detected flames in $30 \mathrm{~cm}$ almost instantaneously.

We also tested and compared several commercial smoke and fire sensors. The first test area was a $4 \mathrm{~m} \times 4 \mathrm{~m}$ small room with a ceiling height of $2.5 \mathrm{~m}$. The test fire was burnt in a $40 \mathrm{~cm} \times 25 \mathrm{~cm}$ barbecue chamber. The test fire was also recorded by an IPX DDK1500 video camera from a distance of $2.5 \mathrm{~m}$. The PIR flame detection sensor system is based on the PARADOX motion detector. The smoke sensors used during the experiments are shown in Fig. 9.

The following sensors were used Flamingo FA11 photo electric smoke sensor, CATACT9451 smoke alarm sensor and CODE DN-23 optical smoke detector. All of the sensors were placed directly above the fire pan. Under the given test conditions, response times for the sensors are given in Table 2. The differential PIR sensor response is better than two smoke detectors even for such small rooms. It is obviously slower than video flame and smoke detectors but they are expensive systems requiring computer processing.

In the second case, the differential PIR sensor system was tested in a large room of size $10 \mathrm{~m} \times 12 \mathrm{~m}$ with a ceiling of $5 \mathrm{~m}$ as shown in Fig. 10. The distance between the $30 \mathrm{~cm}$ diameter pan

\section{Table 1}

Results with 220 fire, 593 non-fire test sequences. The system triggers an alarm when fire is detected within the viewing range of the PIR sensor.

\begin{tabular}{llll}
\hline & $\begin{array}{l}\text { Number of } \\
\text { sequences }\end{array}$ & $\begin{array}{l}\text { Number of false } \\
\text { alarms }\end{array}$ & $\begin{array}{l}\text { Number of } \\
\text { alarms }\end{array}$ \\
\hline $\begin{array}{l}\text { Fire test sequences } \\
\begin{array}{c}\text { Non-fire test } \\
\text { sequences }\end{array}\end{array}$ & 220 & 3 & 217 \\
& 593 & 0 & 0 \\
\hline
\end{tabular}

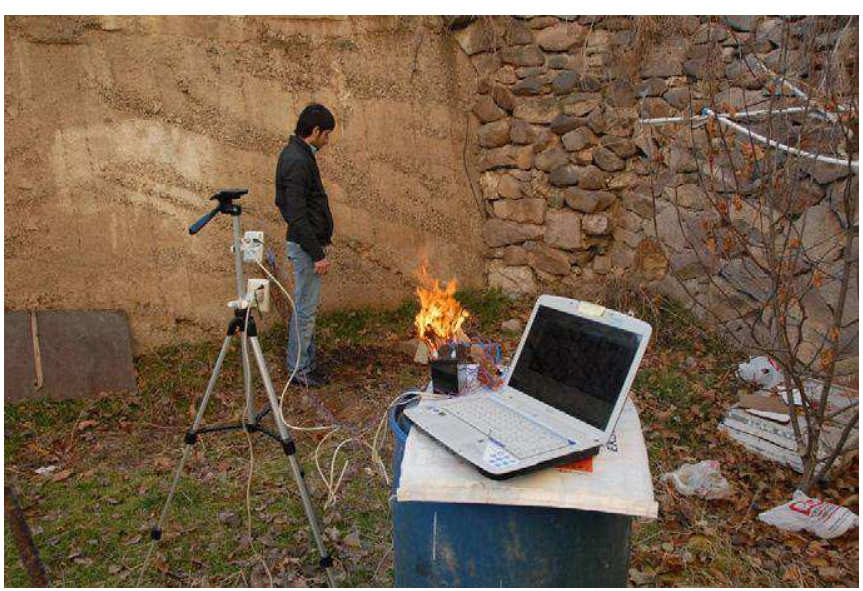

Fig. 8. The PIR sensor is encircled. The fire is close to die out completely. A man is also within the viewing range of the sensor.

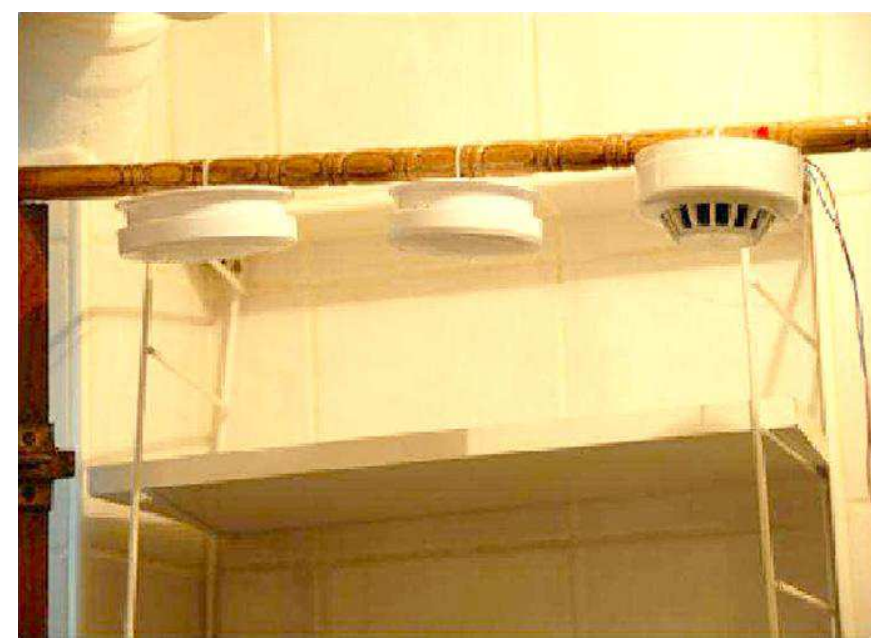

Fig. 9. Smoke sensors setup in a $4 \mathrm{~m} \times 4 \mathrm{~m}$ small room with a ceiling height of $2.5 \mathrm{~m}$

Table 2

Response times of different sensors.

\begin{tabular}{ll}
\hline & Response time \\
\hline Paradox PIR flame sensor & $1 \mathrm{~min} 48 \mathrm{~s}$ \\
Flamingo FA11 photo electric Smoke sensor & $1 \mathrm{~min} 17 \mathrm{~s}$ \\
CATA-CT9451 Smoke alarm Sensor & 9 min $36 \mathrm{~s}$ \\
CODEDN-23 Optical smoke detector & 11 min $32 \mathrm{~s}$ \\
IPXDDK-1500 video camera (as smoke detector) & Less than $10 \mathrm{~s}$ \\
IPXDDK-1500 video camera (as flame detector) & Less than $5 \mathrm{~s}$ \\
\hline
\end{tabular}

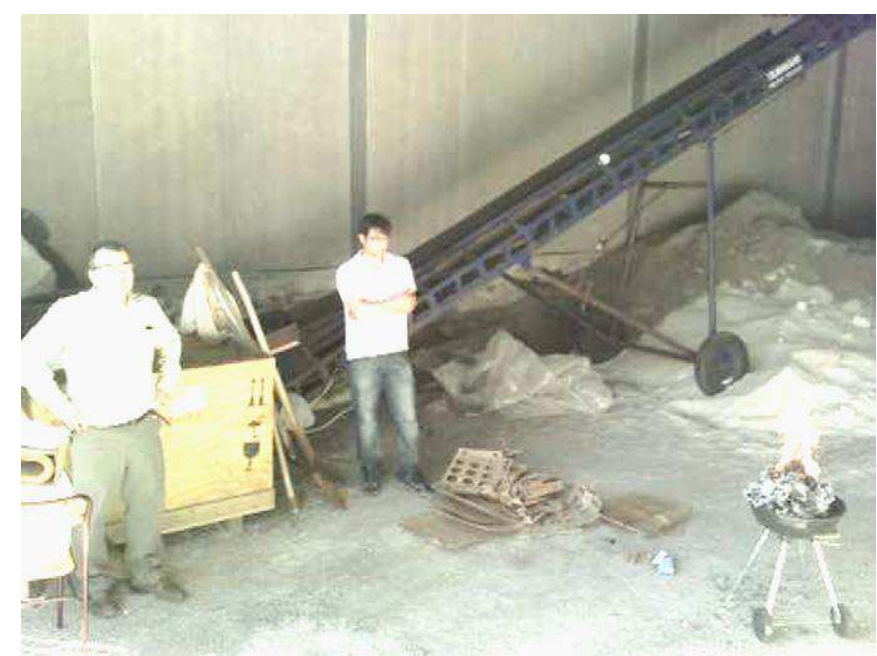

Fig. 10. The PIR sensor is in a large room of size $10 \mathrm{~m} \times 12 \mathrm{~m}$ with a ceiling of $5 \mathrm{~m}$. Cardboard is burned inside the flames.

and the PIR sensor was $9 \mathrm{~m}$. The PIR sensor responded the flames in $35 \mathrm{~s}$ after they became visible. We burned cardboard inside the flames. The response time was less than 2 min after ignition. In this case, none of the smoke sensors could issue an alarm within $10 \mathrm{~min}$.

Finally, we tested the Paradox PIR flame sensor and Flamingo FA11 Photo Electric Smoke Sensor in a $10 \mathrm{~m} \times 9 \mathrm{~m}$ room with a ceiling of $3.5 \mathrm{~m}$. The distance between the sensors and the flame in a barbecue of $40 \mathrm{~cm}$ diameter was $4-6 \mathrm{~m}$. The Flamingo FA11 photo electric smoke sensor could not produce an alarm within 
Table 3

Comparison of the responses of Paradox PIR flame sensor and Flamingo FA11 photo electric smoke sensor to the flame in a barbecue, at 4-6 m distance, in a $10 \mathrm{~m} \times 9 \mathrm{~m} \times 3.5 \mathrm{~m}$ room.

\begin{tabular}{|c|c|c|c|c|}
\hline \multirow{2}{*}{$\begin{array}{l}\text { Fire } \\
\text { area } \\
\left(\mathrm{m}^{2}\right)\end{array}$} & \multirow{2}{*}{$\begin{array}{l}\text { Fire } \\
\text { height } \\
(\mathrm{cm})\end{array}$} & \multirow{2}{*}{$\begin{array}{l}\text { Distance } \\
(\mathrm{m})\end{array}$} & \multicolumn{2}{|l|}{ Alarm time } \\
\hline & & & $\begin{array}{l}\text { Paradox PIR } \\
\text { flame sensor (s) }\end{array}$ & $\begin{array}{l}\text { Flamingo FA11 photo } \\
\text { electric smoke sensor }\end{array}$ \\
\hline 0.04532 & 30 & 4 & 25 & No alarm \\
\hline 0.06265 & 40 & 4 & 19 & No alarm \\
\hline 0.08814 & 65 & 4 & 8 & No alarm \\
\hline 0.09823 & 75 & 4 & 7 & No alarm \\
\hline 0.12150 & 80 & 4 & 5 & No alarm \\
\hline 0.08679 & 90 & 6 & 21 & No alarm \\
\hline
\end{tabular}

$30 \mathrm{~min}$, but the differential PIR sensor responded in a very short time. The results are presented in Table 3.

\section{Conclusion}

In this paper, a differential PIR based flame detection system is proposed. Time-varying analog sensor signal is sampled with a sampling frequency of $50 \mathrm{~Hz}$ and quantized with 8 bits. Markov models corresponding to various human activities and flame flicker process are constructed and trained off-line using the wavelet coefficients of the digitized sensor signal. An event is characterized as an uncontrolled fire when the Markov model corresponding to flame activity produces the highest probability. The proposed algorithm and the system successfully detected flames in all of our experiments.

Comparative results with other sensing modalities are also presented. It is experimentally observed that video based system [22] detects fire and smoke before all the other sensors, but they may produce more false alarms as pointed out in an extensive study by Verstockt [23-26]. In addition, video based systems are significantly more expensive and computationally demanding systems requiring powerful computers to process the video signal. On the other hand, both the equipment and computational cost of the PIR based flame detection system is low. The equipment cost of a differential PIR based flame detection system is low and comparable to the ordinary smoke detectors.

\section{Acknowledgements}

This work is supported in part by the Scientific and Technical Research Council of Turkey and the European Community's Seventh Framework Programme (FP7-ENV-2009-1) under grant agreement no FP7-ENV-244088 "FIRESENSE - Fire Detection and Management through a Multi-Sensor Network for the Protection of Cultural Heritage Areas from the Risk of Fire and Extreme Weather.

\section{References}

[1] B.U. Toreyin, E.B. Soyer, O. Urfalioglu, A.E Cetin, Flame detection using PIR sensors, in: Proceedings of the IEEE 16th Signal Processing, Communication and Applications Conference, SIU 2008, 2008.

[2] C.K. Law, Combustion Physics, Cambridge University Press, 2006.

[3] V. Gubernov, A. Kolobov, A. Polezhaev, H. Sidhu, G. Mercer, Period doubling and chaotic transient in a model of chain-branching combustion wave propagation, Proc. R. Soc. A: Math. Phys. Eng. Sci. 466 (2121) (2010) 2747-2769.

[4] M. Wendeker, J. Czarnigowski, G. Litak, K. Szabelski, Chaotic combustion in spark ignition engines, Chaos Soliton Fract. 18 (2004) 805-808.

[5] P. Manneville, Instabilities, Chaos and Turbulence, Imperial College Press, London, 2004

[6] Fastcom Technology SA, Method and Device for Detecting Fires Based on Image Analysis, PCT Publication. no. WO02/069292, CH-1006, Lausanne, Switzerland, 2002.

[7] B.W. Albers, A.K. Agrawal, Schlieren analysis of an oscillating gas-jet diffusion, Combust. Flame 119 (1999) 84-94.

[8] W. Phillips III., M. Shah, N.V. Lobo, Flame recognition in video, Pattern Recogn. Lett 23 (2002) 319-327.

[9] T. Chen, P. Wu, and Y. Chiou, An early fire-detection method based on image processing, in: Proceedings of the ICIP 2004, 2004, pp. 1707-1710.

[10] B.U. Toreyin, Y. Dedeoglu, U. Gudukbay, A.E. Cetin, Computer vision based system for real-time fire and flame detection, Pattern Recogn. Lett 27 (2006) 49-58.

[11] B.U. Toreyin, Y. Dedeoglu, A.E. Cetin, HMM based falling person detection using both audio and video, in: Proceedings of the IEEE International Workshop on Human-Computer Interaction, Beijing, China, 2005, pp. 211-220.

[12] F. Jabloun, A.E. Cetin, The Teager energy based feature parameters for robust speech recognition in car noise, in: Proceedings of the IEEE ICASSP'99, 1999, pp. 273-276.

[13] H. Bunke, T. Caelli, HMMs Applications in Computer Vision, World Scientific, 2001.

[14] L.R. Rabiner, B.H. Juang, Fundamentals of Speech Recognition, Prentice-Hall Inc., New Jersey, 1993.

[15] M. Thuillard, A new flame detector using the latest research on flames and fuzzy-wavelet algorithms, Fire Saf. J. 37 (2002) 371-380.

[16] M. Thuillard, Wavelets in Soft Computing, World Scientific Publishing Co. Pte Ltd., 2001.

[17] F.C. Carter, N. Cross, Combustion monitoring using infrared array-based detectors, Meas. Sci. Technol. 14 (2003) 1117-1122.

[18] L. Thornqvist, Using a PIR Sensor in a Motion Detection System Design, Future Magazine (accessed 24.12.2011).

[19] C.W. Kim, R. Ansari, A.E. Cetin, A class of linear-phase regular biorthogonal wavelets, in: Proceedings of the IEEE ICASSP'92, 1992, pp. 673-676.

[20] B.U. Toreyin, E.B. Soyer, O. Urfalioglu, A.E. Cetin, Flame detection system based on wavelet analysis of PIR sensor signals with an HMM decision mechanism, in: Proceedings of the EURASIP 16th European Signal Processing Conference (EUSIPCO 2008), 2008.

[21] Kasim Tasdemir Osman Gunay, B. Ugur Toreyin, A. Enis Cetin, Video based wild fire detection at night, Fire Saf. J. 44 (6) (2009) 860-868.

[22] FIRESENSE, Fire Detection and Management Through a Multi-Sensor Network for the Protection of Cultural Heritage Areas from the Risk of Fire and Extreme Weather Conditions, FP7-ENV-2009-1244088-FIRESENSE, 2009, $\langle$ http://www.firesense.eu $\rangle$.

[23] S. Verstockt, Multi-modal Video Analysis for Early Fire Detection, Ph.D. Thesis, Ghent University, November 2011.

[24] S. Verstockt, S. Van Hoecke, N. Tilley, B. Merci, B. Sette, P. Lambert C.F.J. Hollemeersch, R. Van De Walle, 'Hot Topics in Video Fire Surveillance', in Video Surveillance, in: Weiyao Lin (Ed.), InTech, 2011, pp. 443-458 [ISBN: 978-953-307-436-8].

[25] S. Verstockt, C. Poppe, S. Van Hoecke, C. Hollemeersch, B. Merci, B. Sette, P. Lambert, R. Van de Walle, "Silhouette-based multi-sensor smoke detection Coverage analysis of moving object silhouettes in thermal and visual registered images", Machine Vision and Applications (in: press: 'online first').

[26] S. Verstockt, S. Van Hoecke, T. Beji, B. Merci, B. Gouverneur, A. E. Cetin, P. De Potter, R. Van de Walle, "A multi-modal video analysis approach for car park fire detection", Fire Safety Journal (submitted). 